\title{
Perspectives of survivors, families and researchers on key outcomes for research in acute respiratory failure
}

\author{
Victor D Dinglas, ${ }^{1,2}$ Caroline M Chessare, ${ }^{1,2}$ Wesley E Davis, ${ }^{1}$ Ann Parker ${ }_{1,}^{1,2}$ \\ Lisa Aronson Friedman, ${ }^{1,2}$ Elizabeth Colantuoni, ${ }^{1,3}$ Clifton 0 Bingham, ${ }^{4,5,6}$ \\ Alison E Turnbull, ${ }^{1,2,7}$ Dale M Needham ${ }^{1,2,8}$
}

\begin{abstract}
- Additional material is published online only. To view please visit the journal online (http://dx.doi.org/10.1136/ thoraxjn-2017-210234)
\end{abstract}

For numbered affiliations see end of article.

\section{Correspondence to} Victor D Dinglas, Pulmonary and Critical Care Medicine, Johns Hopkins University, $1830 \mathrm{E}$. Monument St, Baltimore, MD 21205, USA; victor.dinglas@ jhmi.edu

Received 8 March 2017 Accepted 19 June 2017 Published Online First 29 July 2017

\section{CrossMark}

To cite: Dinglas VD,

Chessare CM, Davis WE, et al.

Thorax 2018;73:7-12.

\section{ABSTRACT}

Background There is heterogeneity among the outcomes evaluated in studies of survivors of acute respiratory failure (ARF).

Aim To evaluate the importance of specific outcome domains to acute respiratory distress syndrome (ARDS) survivors, their family members and clinical researchers. Methods Nineteen outcome domains were identified from the National Institutes of Health's Patient Reported Outcomes Measurement Information System; WHO's International Classification of Functioning, Disability, and Health; Society of Critical Care Medicine's Post-Intensive Care Syndrome (PICS); as well as patient, clinician and researcher input. We surveyed ARDS survivors, family members and critical care researchers, 279 respondents in total, using a 5-point scale (strongly disagree, disagree, neutral, agree and strongly agree) to rate the importance of measuring each domain in studies of ARF survivors' postdischarge outcomes.

Measurements and main results At least $80 \%$ of patients and family members supported (ie, rated 'agree' or 'strongly agree') that 15 of the 19 domains should be measured in all future studies. Among researchers, 6 of 19 domains were supported, with researchers less supportive for all domains, except survival (95\% vs $72 \%$ support). Overall, four domains were supported by all groups: physical function, cognitive function, return to work or prior activities and mental health.

Conclusion Patient, family and researcher groups supported inclusion of outcome domains that fit within the PICS framework. Patients and family members also supported many additional domains, emphasising the importance of including patients/family, along with researchers, in consensus processes to select core outcome domains for future research studies.

\section{INTRODUCTION}

Survivors of acute respiratory failure (ARF), including those with acute respiratory distress syndrome (ARDS), experience long-term impairments in physical, cognitive and mental health outcomes. $^{1-4}$ Consequently, professional organisations, research funding agencies and clinical researchers have emphasised the importance of measuring patient outcomes beyond short-term survival. ${ }^{5-10}$ An increasing number of studies have evaluated postdischarge outcomes in survivors, although evaluating many different patient outcome domains using many different outcome measures to assess each domain. ${ }^{11}$

\section{Key messages}

What is the key question?

- Among acute respiratory distress syndrome (ARDS) survivors, their family members and clinical researchers, which posthospital outcome domains are considered critical to measure in all research studies of acute respiratory failure (ARF)?

What is the bottom line?

- ARDS survivors, their family members and clinical researchers all support measuring physical function, cognitive function, mental health and return to work or prior activities in all studies of ARF survivors.

Why read on?

- Given the heterogeneity among outcomes reported in studies of ARF, understanding which outcome domains are important to each stakeholder group (patient, family and researchers) is a critical initial step toward creating a minimum set of core outcome measures to evaluate in all future studies.

To address this heterogeneity in the patient outcomes reported in clinical research studies and to facilitate the ability to compare results and synthesise data, creating a 'core outcome set' for clinical research evaluating survivors of ARF has been recommended. ${ }^{12} 13$ A core outcome set is defined as the minimum set of outcome measures always reported in studies within a specific field. ${ }^{14} 15$ An important step toward creating a core outcome set is understanding which outcome domains (ie, outcomes, health conditions or other aspects of health) ${ }^{16}$ are considered important by relevant stakeholders, including patients, family members and researchers. Hence, we surveyed each of these stakeholder groups to obtain their view regarding the importance of measuring specific outcome domains in all clinical studies evaluating ARF patient outcomes after hospital discharge.

\section{METHODS}

\section{Patient and family members}

Patients included in this study were recruited from the national, multicentre Statins for Acutely Injured 
Lungs (SAILS) randomised controlled trial, ${ }^{17}$ after completion of their final 12-month follow-up assessment for the ARDS Network Long-Term Outcomes Study (ALTOS). ${ }^{18}$ To be eligible for inclusion, at the time of this study, patients needed to be alive with a working phone number and able to participate (eg, not incarcerated or hospitalised). Regardless of patient eligibility, family members of ALTOS-SAILS study patients were eligible to participate if they had a working phone number (eg, family members could participate even if the patient had died).

\section{Clinical researchers}

Clinical researchers included in this study were recruited from a pre-existing international database of corresponding authors of 425 articles included in a scoping review of clinical research evaluating intensive care unit (ICU) survivor outcomes after hospital discharge. ${ }^{11}$ This international pool of researchers was invited to complete the survey if they listed an email address in their publication. When a listed email address was outdated, we searched online (eg, search engines, PubMed) and via colleagues to obtain an updated email address.

\section{Survey design}

A total of 19 outcome domains were identified for evaluation in this survey via review of the following patient outcomes frameworks: National Institutes of Health's Patient Reported Outcomes Measurement Information System (PROMIS), ${ }^{19}$ WHO's International Classification of Functioning, Disability, and Health, ${ }^{20}$ and Society of Critical Care Medicine's Post-Intensive Care Syndrome (PICS), ${ }^{8}$ along with specific review and input from patients, clinicians and researchers. Names and descriptions of the 19 domains were adapted for clarity for patients and family members and for internal consistency (eg, consistent use of 'function/symptoms' as part of domain name).

The 19 outcome domains (see online supplementary file 1) were included in a survey instrument to evaluate the level of support for requiring measurement of the domain within a minimum set of domains to be assessed in all studies of posthospital survivorship in ARF patients. The survey was extensively pilot tested with patients, caregivers and clinicians, as well as via two separate modified Delphi Consensus processes conducted with participants at multidisciplinary clinician/researcher conferences held in the USA ( $n=100$ for online survey and $n=44$ for subsequent in-person meeting) and in Australia ( $\mathrm{n}=85$ for in-person meeting). ${ }^{22}$ From this pilot testing, no additional domains were suggested to be added to the list included in the survey instrument for the current study. However, based on this pilot testing, the domain originally called 'Health-related quality of life (Satisfaction with life, personal enjoyment)' was re-named 'Satisfaction with life, or personal enjoyment' to help respondents evaluate the importance of the domain, alone, without considering well-known instruments that measure this domain. In addition, response options were revised from four options ('must always measure', 'sometimes should measure', 'not important to measure' and 'unsure') to five options ('strongly disagree', 'disagree', 'neutral', 'agree' and 'strongly agree').

In addition to the above pilot testing, to ensure completeness of the domains being evaluated, in this survey instrument, patients, family and researchers were specifically asked if any domains were missing from the list of 19 domains they were asked to rate. Moreover, patients and family members were asked whether they would have rated each domain differently if they had been surveyed at 6 months after ICU, rather than the actual timing of the survey administration.
Patient and family surveys were administered via phone, with specific instruction to consider whether each outcome domain was important to be measured in all research studies of critical care survivors. The international cohort of clinical researchers received a similar web-based survey (Qualtrics, Provo, Utah). Completion of the survey indicated informed consent to participate by patients, family members and researchers. The Johns Hopkins Medical Institutions' institutional review board approved this study.

\section{Statistical analyses}

Stacked bar graphs were used to visualise response distribution for all 19 domains (see online supplementary figure 1). A respondent was defined as 'supporting' a domain if he/she selected 'agree' or 'strongly agree' for the domain. For each of the 19 domains, the proportion of respondents who supported the domain was compared for patients versus family members using general estimating equations with binary distribution (logistic regression) adjusting for clustering of patient-family dyads. For two domains, the models would not converge, and a Poisson model was used instead. For patients versus researchers comparison, we used Fisher's exact test. The margin of error for each proportion was calculated at 95\% confidence level. ${ }^{23}$ For patient-family pairs, agreement was evaluated by quantifying the difference in rating within pairs for each outcome domain. The kappa statistic was not appropriate for measuring agreement within this sample because the distribution of actual responses demonstrated symmetrical unbalanced marginal totals that resulted in inappropriately low kappa scores (see online supplementary table 2), as previously described. ${ }^{24}$ Data were analysed using SAS V.9.4 (SAS Institute, Cary, North Carolina, USA), with $\mathrm{p}<0.05$ considered statistically significant.

\section{RESULTS}

\section{Respondent characteristics}

There were 124 patients who were eligible to participate, of whom 15 (12\%) declined to complete the survey, and 31 (25\%) were unable to be interviewed prior to the end of the study, leaving 78 (63\%) who completed the survey. Of the 140 eligible family members, 22 (16\%) declined, and 38 (27\%) were unable to be interviewed prior to the end of the study period, leaving $80(57 \%)$ who completed the survey. Within this cohort, there were 55 patient-family member pairs. On average, the interviews occurred 43 months (SD 10 months) after ICU discharge. Of the 78 survivors who responded, 38\% were male, $86 \%$ were white and $29 \%$ were $\geq 65$ years old (table 1 ). The 80 family member respondents had similar demographic characteristics, and $45 \%$ are spouse/partners and $21 \%$ are adult children (table 1).

Of the 265 researchers who had email addresses in their publication, three did not have working email addresses and could not be found online. Of the 262 researchers emailed, 121 (46\%) responded and completed the survey. Of the 121 respondents, $67 \%$ were male, with 60\% from Europe and 26\% from North America (table 2). The proportions of researchers self-reporting expertise in physical, mental health and cognitive outcomes were $79 \%, 61 \%$ and $52 \%$, respectively.

\section{Patients' perspectives}

Patients generally highly rated the outcome domains, with at least $80 \%$ supporting 15 of the 19 domains. The domains with the highest patient ratings (ie, $>90 \%$ ) were cognitive function and symptoms (96\%), pain (96\%), pulmonary function and 
Table 1 Characteristics of patient and family respondents

\begin{tabular}{lcc}
\hline & $\begin{array}{c}\text { Patient } \\
(\mathbf{n}=78)\end{array}$ & $\begin{array}{c}\text { Family } \\
(\mathbf{n}=80)\end{array}$ \\
\hline Male, $\mathrm{n}(\%)$ & $30(38)$ & $24(30)$ \\
\hline White race, $\mathrm{n}(\%)$ & $67(86)$ & $70(89)$ \\
\hline Age (years), $\mathrm{n}(\%)$ & & \\
\hline $18-24$ & $4(5)$ & $2(3)$ \\
\hline $25-44$ & $15(19)$ & $12(15)$ \\
\hline $45-64$ & $36(46)$ & $41(51)$ \\
\hline $65+$ & $23(29)$ & $25(31)$ \\
\hline Relationship with patient, $\mathrm{n}(\%)$ & & \\
\hline \multicolumn{1}{|c|}{ Partner/spouse } & $\mathrm{N} / \mathrm{A}$ & $36(45)$ \\
\hline \multicolumn{1}{|c|}{ Adult child } & & $17(21)$ \\
\hline \multicolumn{1}{|c|}{ Parent } & & $16(20)$ \\
\hline \multicolumn{1}{|c|}{ Sibling } & & $8(10)$ \\
\hline \multicolumn{1}{|c|}{ Other } & & $3(4)$ \\
\hline Patient has died & $\mathrm{N} / \mathrm{A}$ & $11(14)$ \\
\hline Education in years, mean (SD) & $14(2)$ & $14(2)$ \\
\hline ICU length of stay in days, mean (SD) & $13(10)$ & $13(10)$ \\
\hline Months since ICU discharge, mean (SD) & $43(10)$ & $42(10)$ \\
\hline
\end{tabular}

Missing data: race-1 family.

ICU, intensive care unit; N/A, not applicable.

\begin{tabular}{|c|c|}
\hline & $\mathrm{n}=121$ \\
\hline Male & $81(67)$ \\
\hline \multicolumn{2}{|l|}{ Location } \\
\hline Europe (18 countries) & $72(60)$ \\
\hline North America (two countries) & $32(26)$ \\
\hline Australia/Oceania (two countries) & $12(10)$ \\
\hline Asia (four countries) & $5(4)$ \\
\hline \multicolumn{2}{|l|}{ Research and/or clinical work* } \\
\hline Clinical research & $108(89)$ \\
\hline Clinical work & $71(59)$ \\
\hline Basic or translational research & $30(25)$ \\
\hline Years of experience in clinical research or clinical work, mean (SD) & $13(9)$ \\
\hline \multicolumn{2}{|l|}{ Self-reported subject expertise* } \\
\hline Physical outcomes & $95(79)$ \\
\hline Mental health outcomes & $74(61)$ \\
\hline Cognitive outcomes & $63(52)$ \\
\hline \multicolumn{2}{|l|}{ Clinical training $\dagger$} \\
\hline Physician critical care & $53(75)$ \\
\hline Physician—other & $13(18)$ \\
\hline Physical therapist & $6(8)$ \\
\hline Nurse/nurse practitioner & $2(3)$ \\
\hline Physician—physical medicine and rehabilitation & $2(3)$ \\
\hline Psychologist & $2(3)$ \\
\hline Respiratory therapist & $2(3)$ \\
\hline Other & $1(1)$ \\
\hline
\end{tabular}

*Each respondent can select more than one response.

tEach respondent who has indicated any clinical work $(n=71)$ can select more than one response. symptoms (96\%), fatigue (95\%), physical function and symptoms $(94 \%)$, return to work or prior activities (94\%), mental health conditions and symptoms (92\%) and muscle and/or nerve function (92\%) (table 3; online supplementary figure 1 shows the proportion selecting 'strongly agree' for each domain).

\section{Family members' perspectives}

Similar to patients, family members highly rated the domains, with at least $80 \%$ supporting 16 of the 19 domains. The proportion of patients versus family members who supported a domain was not significantly different for 18 of the 19 domains, with the only significant difference being for the domain of swallowing function and symptoms $(81 \%$ vs $94 \%, p=0.025$; table 3; online supplementary figure 1 shows the proportion selecting 'strongly agree' for each domain). For the 55 patientfamily member pairs, $\geq 80 \%$ of the pairs differed by no more than one response level (eg, one response level, strongly agree vs agree) for 17 of the 19 domains (see online supplementary table 3). The two domains with the lowest proportion of patient-family member pairs within one response level were sexual function and symptoms (65\%) and social roles, activities or relationships (78\%). Notably, when asked if they would have responded differently if the survey was asked 6 months after ICU, $<4 \%$ of patients and family members said 'yes' for all domains.

\section{Researchers' perspectives}

In contrast to patients, researchers' support for measuring outcome domains in all studies was more varied, with $\geq 80 \%$ supporting only 6 of 19 domains. For researchers, the five most highly rated domains were survival (95\%), physical function and symptoms (94\%), cognitive function and symptoms (92\%), return to work or prior activities (92\%), and mental health and symptoms (88\%) (table 3). A larger proportion of patients (vs researchers) supported all domains, except for survival $(72 \%$ vs $95 \%$ supporters, $\mathrm{p}<0.001$ ) (table 3 ; online supplementary figure 1 shows the proportion selecting 'strongly agree' for each domain). Four domains had high ratings by both patients and researchers: physical function and symptoms ( $94 \%$ vs $94 \%$ ), cognitive function and symptoms ( $96 \%$ vs $92 \%)$, return to work or prior activities (94\% vs $92 \%)$, and mental health conditions and symptoms ( $92 \%$ vs $88 \%)$.

Lastly, no new domains were identified after respondents were asked if there was any outcome domain missed from the 19 domains that were presented to them.

\section{DISCUSSION}

This survey of 279 participants included ARDS survivors, their family members and clinical researchers. Patients and family members, in general, rated the 19 outcome domains similarly, with the vast majority supporting to measure 18 of the 19 outcome domains in all survivorship studies. In contrast, researchers generally rated domains less strongly, with the exception of survival. Patients, family members and researchers all reported strong support for measuring the following four outcome domains in all future studies: physical function, cognitive function, return to work or prior activities and mental health.

Both patients and family members rated outcome domains related to cognition, pain, return to work/prior activities, mental health and physical outcomes (fatigue, pulmonary function, muscle/nerve function, physical function) as highly important to assess. These domains are consistent with the evaluations 
Table 3 Patient, family and researcher ratings of the importance of evaluating outcome domains in acute respiratory failure survivorship research*

\begin{tabular}{|c|c|c|c|c|c|}
\hline Domain questions & $\begin{array}{l}\text { Patients } \\
(\mathrm{n}=78)\end{array}$ & $\begin{array}{l}\text { Family } \\
(n=80)\end{array}$ & $\begin{array}{l}\text { Researchers } \\
(n=121)\end{array}$ & $\begin{array}{l}\text { Patient vs family, } \\
\text { p valuet }\end{array}$ & $\begin{array}{l}\text { Patient vs researcher, } \\
\text { p value } \neq\end{array}$ \\
\hline Cognitive function and symptoms & $75(96 \% \pm 4 \%)$ & $80(100 \% \pm 0 \%)$ & $111(92 \% \pm 5 \%)$ & 0.083 & 0.256 \\
\hline Pain & $75(96 \% \pm 4 \%)$ & $78(98 \% \pm 3 \%)$ & $101(83 \% \pm 7 \%)$ & 0.623 & 0.006 \\
\hline Pulmonary function and symptoms & $75(96 \% \pm 4 \%)$ & $80(100 \% \pm 0 \%)$ & $87(72 \% \pm 8 \%)$ & 0.083 & $<0.001$ \\
\hline Fatigue & $74(95 \% \pm 5 \%)$ & $76(95 \% \pm 5 \%)$ & $92(76 \% \pm 8 \%)$ & 0.958 & $<0.001$ \\
\hline Physical function and symptoms & $73(94 \% \pm 5 \%)$ & $79(99 \% \pm 2 \%)$ & $114(94 \% \pm 4 \%)$ & 0.117 & 1.000 \\
\hline Return to work or prior activities & $73(94 \% \pm 5 \%)$ & $74(93 \% \pm 6 \%)$ & $111(92 \% \pm 5 \%)$ & 0.782 & 0.786 \\
\hline Mental health conditions and symptoms & $72(92 \% \pm 6 \%)$ & $74(93 \% \pm 6 \%)$ & $106(88 \% \pm 6 \%)$ & 0.962 & 0.350 \\
\hline Muscle and/or nerve function & $72(92 \% \pm 6 \%)$ & $79(99 \% \pm 2 \%)$ & $93(77 \% \pm 8 \%)$ & 0.083 & 0.006 \\
\hline Healthcare resource utilisation & $70(90 \% \pm 7 \%)$ & $75(94 \% \pm 5 \%)$ & $84(69 \% \pm 8 \%)$ & 0.387 & $<0.001$ \\
\hline Type of residence & $70(90 \% \pm 7 \%)$ & $69(86 \% \pm 8 \%)$ & $91(75 \% \pm 8 \%)$ & 0.502 & 0.016 \\
\hline Impact on family and/or caregivers & $69(88 \% \pm 7 \%)$ & $73(91 \% \pm 6 \%)$ & $84(69 \% \pm 8 \%)$ & 0.547 & 0.002 \\
\hline Sleep function and symptoms & $69(88 \% \pm 7 \%)$ & $77(96 \% \pm 4 \%)$ & $85(70 \% \pm 8 \%)$ & 0.085 & 0.003 \\
\hline Satisfaction with life or personal enjoyment & $68(87 \% \pm 7 \%)$ & $68(85 \% \pm 8 \%)$ & $92(76 \% \pm 8 \%)$ & 0.695 & 0.067 \\
\hline Gastrointestinal function and symptoms & $64(82 \% \pm 9 \%)$ & $74(93 \% \pm 6 \%)$ & $39(32 \% \pm 8 \%)$ & 0.051 & $<0.001$ \\
\hline Swallowing function and symptoms & $63(81 \% \pm 9 \%)$ & $75(94 \% \pm 5 \%)$ & $58(48 \% \pm 9 \%)$ & 0.025 & $<0.001$ \\
\hline Financial impact on patient & $58(74 \% \pm 10 \%)$ & $59(74 \% \pm 10 \%)$ & $61(50 \% \pm 9 \%)$ & 0.748 & 0.001 \\
\hline Social roles, activities or relationships & $57(73 \% \pm 10 \%)$ & $58(73 \% \pm 10 \%)$ & $86(71 \% \pm 8 \%)$ & 0.906 & 0.872 \\
\hline Survival & $56(72 \% \pm 10 \%)$ & $66(83 \% \pm 8 \%)$ & $115(95 \% \pm 4 \%)$ & 0.106 & $<0.001$ \\
\hline Sexual function and symptoms & $39(50 \% \pm 11 \%)$ & $36(45 \% \pm 11 \%)$ & $53(44 \% \pm 9 \%)$ & 0.489 & 0.467 \\
\hline
\end{tabular}

*Count (\% \pm margin of error at $95 \%$ confidence level) of participants who selected 'agree' or 'strongly agree' on a 5-point Likert scale ranging from 'strongly agree' to 'strongly disagree'. All respondents completed rating for all 19 domains.

tp value calculated using general estimating equations with binary distribution (logistic model). For 'Cognitive function and symptoms' and 'Pulmonary function and symptoms,' the models did not converge using logistic model; hence, a Poisson model was used

łp value calculated using Fisher's test

and impairments reported in ICU survivorship studies, ${ }^{2} 2526$ including in a recent systematic review of qualitative studies of ICU survivors, ${ }^{27}$ and a recent qualitative study of posthospital patient outcomes specifically in ARF and ARDS survivors. ${ }^{28}$ Patient-family member pairs were generally within one rating level from each other, highlighting similarity in their views. To our knowledge, no prior investigations have surveyed both ARDS survivors and family members regarding which posthospitalisation outcome domains they consider important to study. In other studies, for example, with quality of life, inter-rater agreement between patient and family has ranged from poor to excellent in different studies. ${ }^{29-33}$ Additionally, family members are generally more accurate than clinicians at predicting patients' treatment preferences. $^{3435}$ However, our study aimed to describe each of the stakeholder group's perspective, and we did not ask family members or researchers to respond in the way that they think the patient would have responded. The latter technique ${ }^{3637}$ is often used in studies that have the intent of specifically comparing inter-rater agreement, which was not the specific intent of our study.

Researchers differed from patients and their family members in the strength of their support for 12 of the 19 outcome domains. Of note, survival was the domain with the highest proportion of researchers rating 'agree' or 'strongly agree', whereas this domain received the second lowest ratings from patients. These patients may underestimate or be unaware of the high risk of mortality in the years immediately after critical illness. This difference may reflect that only surviving patients could be included in this survey. However, the number of years since their ICU discharge was not associated with the proportion of patients supporting the survival domain. Researchers may have ranked survival highly because they recognised the importance of understanding the competing risk of death when assessing all other patient outcomes in clinical research studies. ${ }^{38} 39$ Additionally, valuing survival is consistent with previous critical care research that has traditionally used mortality as a primary outcome. ${ }^{40}$

After the survival domain, the four domains that garnered the next highest support from researchers were physical function, cognitive function, mental health and return to work or prior activities, which were rated similarly by patients and family members. These four domains fit within the PICS conceptual framework. ${ }^{8} 2141$ Furthermore, these four domains, along with survival, were highly rated by clinicians in two recent international, in-person consensus meetings that evaluated core outcome domains for ICU survivors. ${ }^{22}$ The only outcome domain that was strongly supported in this prior research ${ }^{19}$ that was not highly rated in the present study was health-related quality of life, which was renamed as 'satisfaction with life, or personal enjoyment' in the present study (as described in the Methods section). The change in labelling may explain this difference in results.

Gaining consensus for a common set of outcome domains that are measured in all long-term outcomes studies in ARF survivors will help advance survivorship research by reducing heterogeneity in outcomes measured ${ }^{11}$ and decreasing bias from potential selective outcome reporting. ${ }^{42}{ }^{43}$ Moreover, findings of this study emphasise the importance of patient and family input to decision making regarding core outcome domains, which has been under-represented in prior projects aimed at creating core outcome sets. ${ }^{44}$ Incorporating patient perspectives is important to ensure that the outcomes measured by researchers are patient centred. ${ }^{45} 46$ Given the similarity in how ARDS survivors and their family caregivers view these outcome domains in 
the present study, including family members in the process of selecting a minimum set of outcome domains may be a feasible way to boost patient representation.

This study has several strengths including surveying a large number of patients and family members from across the USA and clinical researchers from around the world to provide a geographically diverse sample. There are also some potential limitations. First, patients and family members were interviewed at a mean (SD) of 43 (10) months after critical illness, and results may have been different at an earlier time point. However, when asked if they would respond differently if reflecting on their status at 6 months after ICU discharge, $<4 \%$ responded that they would have answered differently for each of the 19 domains. Second, only approximately $50 \%$ of patients, family members and researchers responded to the survey giving rise to possible selection bias in the reported results. Additionally, there could be a potential bias related to presenting domains in a fixed order to participants. However, there were no missing data for all 19 domains for all respondents, which may help minimise this concern. Lastly, patients were exclusively ARDS survivors, enrolled in a randomised control trial and survived beyond 3 years after ARDS; hence, patients and family perspectives may not be generalisable to other ARDS cohorts, to ARF patients or to other critically ill patients.

In conclusion, patients and family members agreed that studies evaluating their outcomes after hospital discharge should measure many domains. Despite differences between patients and researchers' perspectives on the relative importance of various outcome domains, the domains with agreement across all stakeholder groups (physical function, cognitive function, return to work or prior activities and mental health) generally encompassed the existing PICS framework. Given different perspectives of patients/families and researchers, participation of both these stakeholder groups in formal consensus process is important to ensure the development of a patient-centred set of core outcome domains.

\section{Author affiliations}

${ }^{1}$ Outcomes After Critical Illness and Surgery Group, Johns Hopkins University, Baltimore, Maryland, USA

${ }^{2}$ Division of Pulmonary and Critical Care Medicine, School of Medicine, Johns Hopkins University, Baltimore, Maryland, USA

${ }^{3}$ Department of Biostatistics, Bloomberg School of Public Health, Johns Hopkins University, Baltimore, Maryland, USA

${ }^{4}$ Johns Hopkins Arthritis Center, Johns Hopkins University, Baltimore, Maryland, USA ${ }^{5}$ Rheumatic Diseases Research Core Center, Johns Hopkins University, Baltimore, Maryland, USA

${ }^{6}$ Divisions of Rheumatology and Allergy and Clinical Immunology, Johns Hopkins University, Baltimore, Maryland, USA

${ }^{7}$ Department of Epidemiology, Bloomberg School of Public Health, Johns Hopkins University, Baltimore, Maryland, USA

${ }^{8}$ Department of Physical Medicine and Rehabilitation, School of Medicine, Johns Hopkins University, Baltimore, Maryland, USA

Contributors VDD, AET and DMN contributed to conception and design of the manuscript. VDD, LAF, EC, AET, COB and DMN contributed to analysis and interpretation of data. VDD drafted the manuscript, and all other authors critically revised it for important intellectual content. All authors gave final approval of the manuscript version to be published.

Funding This research was supported by the National Heart, Lung, and Blood Institute (grant number R24HL111895). AP was supported by KL2 Mentored Career Development Award grant number (4KL2TR001077-04).

Disclaimer The funding bodies had no role in the study design; in the collection, analysis and interpretation of data; in the writing of the report; and in the decision to submit the manuscript for publication.

Competing interests None declared.

Ethics approval Johns Hopkins University Institutional Review Board.
Provenance and peer review Not commissioned; externally peer reviewed.

(c) Article author(s) (or their employer(s) unless otherwise stated in the text of the article) 2018. All rights reserved. No commercial use is permitted unless otherwise expressly granted.

\section{REFERENCES}

1 Needham DM, Colantuoni E, Dinglas VD, et al. Rosuvastatin versus placebo for delirium in intensive care and subsequent cognitive impairment in patients with sepsis-associated acute respiratory distress syndrome: an ancillary study to a randomised controlled trial. Lancet Respir Med 2016;4:203-12.

2 Herridge MS, Cheung AM, Tansey CM, et al. One-year outcomes in survivors of the acute respiratory distress syndrome. N Engl J Med 2003;348:683-93.

3 Huang M, Parker AM, Bienvenu OJ, et al. Psychiatric symptoms in acute respiratory distress syndrome survivors: a 1-year national multicenter study. Crit Care Med 2016:44:954-65.

4 Fan E, Dowdy DW, Colantuoni E, et al. Physical complications in acute lung injury survivors: a two-year longitudinal prospective study. Crit Care Med 2014;42:849-59.

5 Angus DC, Mira JP, Vincent JL. Improving clinical trials in the critically ill. Crit Care Med 2010;38:527-32.

6 Angus DC, Carlet J. 2002 Brussels Roundtable Participants. Surviving intensive care: a report from the 2002 Brussels Roundtable. Intensive Care Med 2003;29:368-77.

7 Spragg RG, Bernard GR, Checkley W, et al. Beyond mortality: future clinical research in acute lung injury. Am J Respir Crit Care Med 2010;181:1121-7.

8 Needham DM, Davidson J, Cohen $\mathrm{H}$, et al. Improving long-term outcomes after discharge from intensive care unit: report from a stakeholders' conference. Crit Care Med 2012;40:502-9.

9 Deutschman CS, Ahrens T, Cairns CB, et al. Multisociety Task Force for Critical Care Research: key issues and recommendations. Crit Care Med 2012;40:254-60.

10 Carson SS, Goss CH, Patel SR, et al. An official American Thoracic Society research statement: comparative effectiveness research in pulmonary, critical care, and sleep medicine. Am J Respir Crit Care Med 2013;188:1253-61.

11 Turnbull AE, Rabiee A, Davis WE, et al. Outcome measurement in ICU survivorship research from 1970 to 2013: a scoping review of 425 publications. Crit Care Med 2016:44:1267-77.

12 Blackwood B, Marshall J, Rose L. Progress on core outcome sets for critical care research. Curr Opin Crit Care 2015;21:439-44.

13 Needham DM. Understanding and improving clinical trial outcome measures in acute respiratory failure. Am J Respir Crit Care Med 2014;189:875-7.

14 Clarke M. Standardising outcomes for clinical trials and systematic reviews. Trials 2007:8:39.

15 Williamson PR, Altman DG, Blazeby JM, et al. Developing core outcome sets for clinical trials: issues to consider. Trials 2012:13:132.

16 Boers M, Kirwan JR, Wells G, et al. Developing core outcome measurement sets for clinical trials: OMERACT filter 2.0. J Clin Epidemiol 2014;67:745-53.

17 Truwit JD, Bernard GR, Steingrub J, et al. Rosuvastatin for sepsis-associated acute respiratory distress syndrome. N Engl J Med 2014;370:2191-200.

18 Dinglas VD, Hopkins RO, Wozniak AW, et al. One-year outcomes of rosuvastatin versus placebo in sepsis-associated acute respiratory distress syndrome: prospective followup of SAILS randomised trial. Thorax 2016:71:401-10.

19 Cella D, Riley W, Stone A, et al. The Patient-Reported Outcomes Measurement Information System (PROMIS) developed and tested its first wave of adult selfreported health outcome item banks: 2005-2008. J Clin Epidemiol 2010;63:1179-94.

20 World Health Organization. International classification of functioning, disability and Health. Geneva, Switzerland: World Health Organization, 2001

21 Elliott D, Davidson JE, Harvey MA, et al. Exploring the scope of post-intensive care syndrome therapy and care: engagement of non-critical care providers and survivors in a second stakeholders meeting. Crit Care Med 2014;42:2518-26.

22 Hodgson CL, Turnbull AE, Iwashyna TJ, et al. Core domains in evaluating patient outcomes after acute respiratory failure: international multidisciplinary clinician consultation. Phys Ther 2017;97:167-74.

23 Rubenfeld GD. Surveys: an introduction. Respir Care 2004;49:1181-5.

24 Feinstein AR, Cicchetti DV. High agreement but low kappa: i. the problems of two paradoxes. J Clin Epidemio/ 1990;43:543-9.

25 Desai SV, Law TJ, Needham DM. Long-term complications of critical care. Crit Care Med 2011;39:371-9.

26 Girard TD, Dittus RS, Ely EW. Critical illness brain injury. Annu Rev Med 2016:67:497-513.

27 Hashem MD, Nallagangula A, Nalamalapu S, et al. Patient outcomes after critical illness: a systematic review of qualitative studies following hospital discharge. Crit Care 2016;20:345.

28 Eakin MN, Patel Y, Mendez-Tellez P, et al. Patients' outcomes after acute respiratory failure: $\mathrm{A}$ qualitative study with the promis framework. Am J Crit Care 2017:26:456-65.

29 Dinglas VD, Gifford JM, Husain N, et al. Quality of life before intensive care using EQ5D: patient versus proxy responses. Crit Care Med 2013;41:9-14.

30 Gifford JM, Husain N, Dinglas VD, et al. Baseline quality of life before intensive care: a comparison of patient versus proxy responses. Crit Care Med 2010;38:855-60. 
31 Scales DC, Tansey CM, Matte A, et al. Difference in reported pre-morbid health-related quality of life between ARDS survivors and their substitute decision makers. Intensive Care Med 2006;32:1826-31.

32 Rogers J, Ridley S, Chrispin P, et al. Reliability of the next of kins' estimates of critically ill patients' quality of life. Anaesthesia 1997;52:1137-43.

33 Elliott D, Lazarus R, Leeder SR. Proxy respondents reliably assessed the quality of life of elective cardiac surgery patients. J Clin Epidemio/ 2006;59:153-9.

34 Coppola KM, Ditto PH, Danks JH, et al. Accuracy of primary care and hospital-based physicians' predictions of elderly outpatients' treatment preferences with and without advance directives. Arch Intern Med 2001;161:431-40.

35 Downey L, Au DH, Curtis JR, et al. Life-sustaining treatment preferences: matches and mismatches between patients' preferences and clinicians' perceptions. J Pain Symptom Manage 2013;46:9-19.

36 McPhail S, Beller E, Haines T. Two perspectives of proxy reporting of health-related quality of life using the Euroqol-5D, an investigation of agreement. Med Care 2008;46:1140-8.

37 Pickard AS, Knight SJ. Proxy evaluation of health-related quality of life: a conceptua framework for understanding multiple proxy perspectives. Med Care 2005;43:493-9.

38 Wolkewitz M, Cooper BS, Bonten MJ, et al. Interpreting and comparing risks in the presence of competing events. BMJ 2014;349:95060
39 van Walraven C, McAlister FA. Competing risk bias was common in Kaplan-Meier risk estimates published in prominent medical journals. J Clin Epidemio/ 2016;69:170-3.

40 Brinkman S, Bakhshi-Raiez F, Abu-Hanna A, et al. Determinants of mortality after hospital discharge in ICU patients: literature review and Dutch cohort study. Crit Care Med 2013;41:1237-51.

41 Harvey MA, Davidson JE. Postintensive care syndrome: right care, right now... and later. Crit Care Med 2016;44:381-5.

42 Dwan K, Altman DG, Arnaiz JA, et al. Systematic review of the empirical evidence of study publication bias and outcome reporting bias. PLoS One 2008;3:e3081.

43 Kirkham JJ, Dwan KM, Altman DG, et al. The impact of outcome reporting bias in randomised controlled trials on a cohort of systematic reviews. BMJ 2010;340:c365.

44 Gargon E, Gurung B, Medley N, et al. Choosing important health outcomes for comparative effectiveness research: a systematic review. Value Health 2014;17:A435

45 Tunis SR, Clarke M, Gorst SL, et al. Improving the relevance and consistency of outcomes in comparative effectiveness research. J Comp Eff Res 2016:5:193-205.

46 Young B, Bagley H. Including patients in core outcome set development: issues to consider based on three workshops with around 100 international delegates. Research Involvement and Engagement 2016;2:25 